\title{
On Renewable Sensor Networks with Wireless Energy Transfer: The Multi-Node Case
}

\author{
Liguang Xie \\ Yi Shi \\ Y. Thomas Hou* \\ Wenjing Lou \\ Hanif D. Sherali \\ Scott F. Midkiff \\ Virginia Polytechnic Institute and State University, USA
}

\begin{abstract}
Wireless energy transfer based on magnetic resonant coupling is a promising technology to replenish energy to sensor nodes in a wireless sensor network (WSN). However, charging sensor node one at a time poses a serious scalability problem. Recent advances in magnetic resonant coupling shows that multiple nodes can be charged at the same time. In this paper, we exploit this multi-node wireless energy transfer technology to address energy issue in a WSN. We consider a wireless charging vehicle (WCV) periodically traveling inside a WSN and charging sensor nodes wirelessly. We propose a cellular structure that partitions the two-dimensional plane into adjacent hexagonal cells. The WCV visits these cells and charge sensor nodes from the center of a cell. We pursue a formal optimization framework by jointly optimizing traveling path, flow routing and charging time. By employing discretization and a novel ReformulationLinearization Technique (RLT), we develop a provably nearoptimal solution for any desired level of accuracy.
\end{abstract}

\section{INTRODUCTION}

Wireless energy transfer based on magnetic resonant coupling is widely regarded as a breakthrough technology in our time [9]. By having magnetic resonant coils operating at the same resonant frequency, Kurs et al. demonstrated that energy could be transferred efficiently from one source coil to one receiver coil via nonradiative electromagnetic field (without any physical contact, i.e., wirelessly). ${ }^{1}$ What makes such wireless energy transfer technology particularly attractive is that it does not require line-of-sight (LOS) or any alignment (i.e., omnidirectional), and is insensitive to the neighboring environment. Since its inception, magnetic resonant coupling has quickly found commercial applications (see, e.g., [12], [15], [22]).

In [17], we first applied this technology for a wireless sensor network (WSN) and showed that through periodic wireless energy transfer, a WSN could remain operational forever, i.e., infinite lifetime. Specifically, we showed that by having a wireless charging vehicle (WCV) visit each sensor node in the network and charge it periodically, one can ensure that each sensor node never runs out of energy.

An open problem in [17] is scalability. That is, as the node density increases in a WSN, how can a WCV ensure that each node is charged in a timely manner without running out

For correspondence, please contact Prof. Y.T. Hou, Dept. of Electrical and Computer Engineering, 302 Whittemore Hall, MC 0111, Virginia Tech, Blacksburg, VA 24061, USA. Email: thou@vt.edu.

${ }^{1}$ It is important to note that magnetic resonant coupling is different from radiative energy transfer [14], [16]. The latter has much lower energy transfer efficiency. of energy? The technology developed in [9] was limited to charging one node at a time and is not scalable as network node density increases.

Kurs et al. also recognized this problem and recently developed an enhanced technology (by properly tuning coupled resonators) that allows energy to be transferred to multiple receiving nodes simultaneously [10]. They also showed that the overall output efficiency of charging multiple devices was larger than the output efficiency of charging each device individually.

Inspired by this new advance in wireless energy transfer, in this paper, we explore how such multi-node charging technology can address the scalability problem in charging a WSN. Following the setting in [17], we consider a WCV periodically traveling inside the network and charging sensor nodes at different stops along its traveling path. Upon completing each trip, the WCV returns to its home service station, takes a "vacation", and starts out for its next trip cycle. In contrast to [17], the WCV is now capable of charging multiple nodes at the same time, as long as these nodes are within its charging range. Under this setting, we ask the following fundamental question: Can such multi-node charging technology address the scalability problem in a dense WSN?

To answer this question, we take a formal optimization approach in this study. Based on the charging range of a WCV, we propose a cellular structure that partitions the twodimensional plane into adjacent hexagonal cells (similar to cellular structure for cellular telecommunications). To charge the sensor nodes in a cell, the WCV only needs to visit the center of the cell. Based on a general energy charging model, we formulate a joint optimization problem for traveling path, flow routing and charging time, with the objective of maximizing the ratio of the WCV's vacation time (time spent at its home service station) over the cycle time. We show that our optimization problem is a nonlinear program (NLP) and is NP-hard in general. By employing discretization and a novel Reformulation-Linearization Technique (RLT), we develop a provably near-optimal solution for any desired level of accuracy. Using numerical results, we show that our solution can effectively address the charging scalability problem in a dense WSN.

The rest of this paper is organized as follows. In Section II, we describe the mathematical model in our study. Section III presents a formulation of our optimization problem and discusses several interesting properties associated with an optimal 


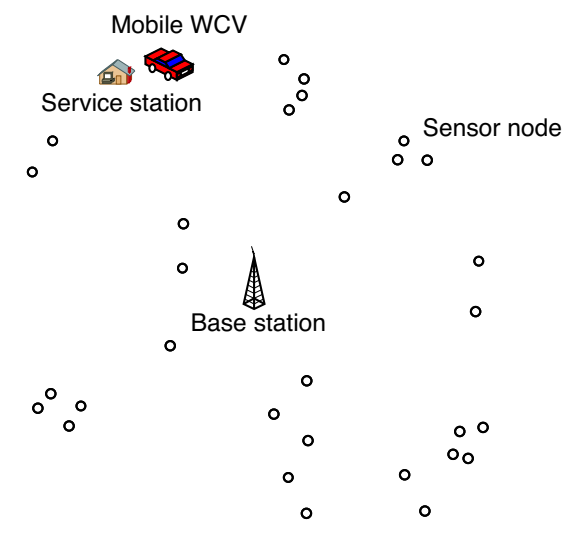

Fig. 1. An example sensor network with a mobile WCV.

solution. In Section IV, we develop a near-optimal solution. In Section V, we present numerical results to demonstrate our solution. Section VI concludes this paper.

\section{Mathematical Modeling}

\section{A. Cellular Structure and Energy Charging Behavior}

We consider a set of sensor nodes $\mathcal{N}$ distributed over a twodimensional area (see Fig. 1). Each sensor node has a battery with a capacity of $E_{\max }$ and is fully charged initially. Also, denote $E_{\min }$ as the minimum level of energy at a battery for it to be operational. Each sensor node $i$ generates sensing data with a rate $R_{i}$ (in $\mathrm{b} / \mathrm{s}$ ), $i \in \mathcal{N}$. Within the sensor network, there is a fixed base station $(B)$, which is the sink node for all data generated by all sensor nodes. Multi-hop data routing is employed for forwarding all data streams to the base station.

To recharge the battery at each sensor node, a WCV is employed. The WCV starts at the service station $(S)$, and travels (at speed of $V \mathrm{~m} / \mathrm{s}$ ) to various spots inside the network to charge sensor nodes. As discussed, the WCV can charge multiple nodes simultaneously as long as they are within its charging range, denoted as $D$. The charging range $D$ is chosen such that the power reception rate at a sensor node is at least over a threshold (denoted as $\delta$ ) when it is within this range from the WCV. The power reception rate at a sensor node $i$, denoted as $U_{i}$, is a distance-dependent parameter, and decreases with the distance between itself and the WCV. When a sensor node is more than a distance of $D$ away from the $\mathrm{WCV}$, we assume its power reception rate is too low to make magnetic resonant coupling work properly at a sensor node battery.

Based on this model, we partition the two-dimensional plane with hexagonal cells with side length of $D$ (see Fig. 2). To charge sensor nodes in a cell, the WCV only needs to visit the center of a cell, as all sensor nodes within a hexagonal cell are within a distance of $D$ from the cell center. ${ }^{2}$ For tractability

\footnotetext{
${ }^{2}$ We ignore the "edge effect" where a sensor node residing outside the cell but inside the circle with a radius of $D$ can still be charged from this cell. Note that such omission of over-charging will not affect the feasibility of our solution.
}

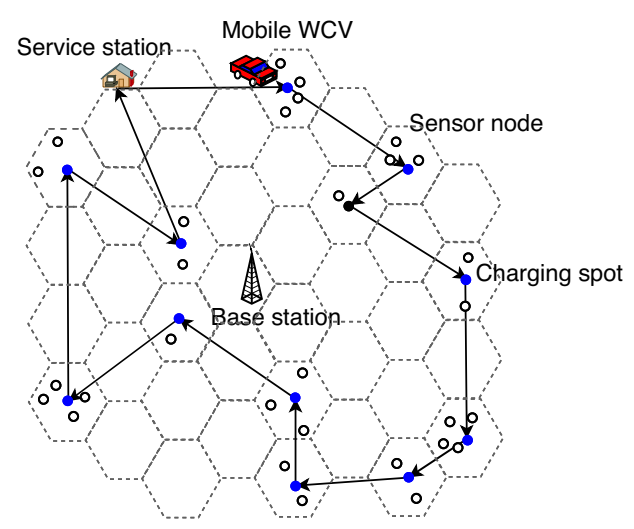

Fig. 2. An example sensor network with a mobile WCV. Solid dots represent cell centers and empty circles represents sensor nodes.

in our analysis, we assume that the WCV will only visit the center of a cell.

Under the cellular structure, denote $D_{i}$ the distance from node $i$ to its cell center. Then nodes $i$ 's power reception rate is $U_{i}=\mu\left(D_{i}\right) \cdot U_{\text {Full }}$, where $U_{\text {Full }}$ is the full output power from WCV for a single sensor node and $\mu\left(D_{i}\right)$ is the efficiency of wireless power transfer. Note that $\mu\left(D_{i}\right)$ is a decreasing function of $D_{i}$ and $0 \leq \mu\left(D_{i}\right) \leq 1$.

Under this setting, we are interested in finding out how the WCV should travel among these cell centers so that (i) none of the sensor nodes will ever run out of energy, and (ii) some performance objective can be optimized. In the rest of this section, we present mathematical characterization of the WCV's traveling path and cycle time (Section II-B), data flow routing and energy consumption model (Section II-C), and energy dynamics at a sensor node (Section II-D).

\section{B. WCV Traveling Path and Cycle Time}

Denote $\mathcal{Q}$ the set of hexagonal cells containing at least one sensor node (see Fig. 3). Re-index these cells in $\mathcal{Q}$ as $k=$ $1,2, \cdots,|\mathcal{Q}|$ and denote $\mathcal{N}_{k}$ the set of sensor nodes in the $k$ th hexagonal cell. Then $\mathcal{N}=\bigcup_{k \in \mathcal{Q}} \mathcal{N}_{k}$.

Denote $\tau_{k}$ the time span for the WCV to be present at the center of cell $k \in \mathcal{Q}$. Throughout $\tau_{k}$, the WCV re-charges all sensor nodes within this hexagonal cell simultaneously via multi-node charging technology [10]. After $\tau_{k}$, the WCV leaves the current cell and travels to the next cell on its path. In our formulation, we assume that the WCV visits a cell only once during a cycle. Denote $P=\left(\pi_{0}, \pi_{1}, \ldots, \pi_{|\mathcal{Q}|}, \pi_{0}\right)$ the physical path traversed by the WCV during a cycle, which starts from and ends at the service station (i.e., $\pi_{0}=S$ ) and the $k$ th cell traversed by the WCV along path $P$ is $\pi_{k}$, $1 \leq k \leq|\mathcal{Q}|$. Denote $D_{P}$ the physical distance of path $P$ and $\tau_{P}=D_{P} / V$ the time spent for traveling over distance $D_{P}$.

After the WCV visits all $|\mathcal{Q}|$ cells in the network, it will return to its service station to be serviced (e.g., replacing its battery, maintenance service, vacation) before the next trip. We call this resting period vacation time, denoted as $\tau_{\text {vac }}$. After this vacation, the WCV will go out for its next trip. Denote 


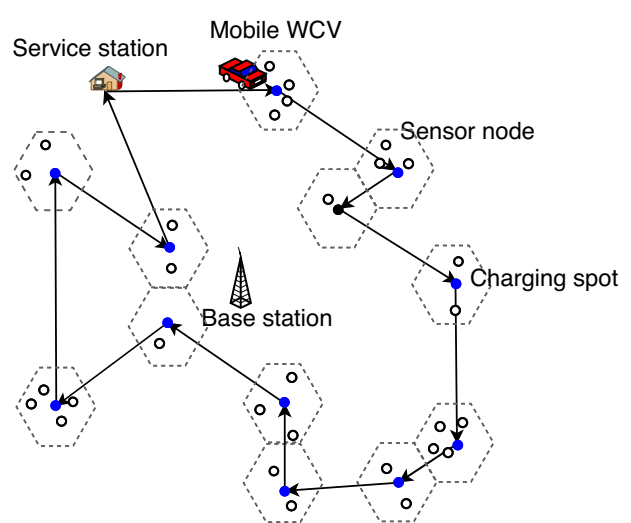

Fig. 3. An example sensor network with a mobile WCV. Only those cells with sensor nodes are shown in this figure.

the time of a traveling cycle of the WCV as $\tau$. Then this cycle time $\tau$ can be written as

$$
\tau=\tau_{P}+\tau_{\text {vac }}+\sum_{k \in \mathcal{Q}} \tau_{k},
$$

where $\sum_{k \in \mathcal{Q}} \tau_{k}$ is the total amount of time the WCV spends for battery charging. We assume that the WCV has sufficient energy to charge all sensor nodes before returning to its service station.

In this setting, a number of performance objectives can be considered. Similar to our work for single-node charging in [17], we study how to maximize the percentage of time in a cycle that the WCV can take vacation (i.e., $\frac{\tau_{\text {vac }}}{\tau}$ ). In practice, this is equivalently to minimizing the percentage of time that the WCV is out in the field.

\section{Data Flow Routing and Energy Consumption}

To model multi-hop data routing, denote $f_{i j}$ and $f_{i B}$ the flow rates from sensor node $i$ to sensor node $j$ and the base station $B$, respectively. Then we have the following flow balance constraint at each sensor node $i$.

$$
\sum_{k \in \mathcal{N}}^{k \neq i} f_{k i}+R_{i}=\sum_{j \in \mathcal{N}}^{j \neq i} f_{i j}+f_{i B} \quad(i \in \mathcal{N}) .
$$

Although both flow routing and flow rates are part of our optimization problem, we assume they are independent of time.

In this paper, we use the following energy consumption model at each sensor node [7]. To transmit a flow rate of $f_{i j}$ from node $i$ to node $j$, the transmission power is $C_{i j} \cdot f_{i j}$, where $C_{i j}$ is the rate of energy consumption for transmitting one unit of data rate from node $i$ to node $j$, and is modeled as

$$
C_{i j}=\beta_{1}+\beta_{2} D_{i j}^{\alpha} .
$$

$D_{i j}$ is the distance between nodes $i$ and $j, \beta_{1}$ is a distanceindependent constant term, $\beta_{2}$ is a coefficient of the distancedependent term and $\alpha$ is the path loss index. Similarly, denote $C_{i B}$ as the rate of energy consumption for transmitting one unit of data rate from node $i$ to the base station $B$. Then the

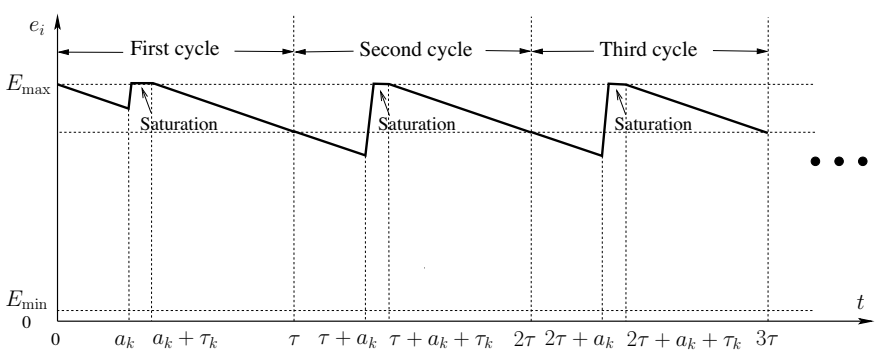

Fig. 4. The energy level of node $i \in \mathcal{N}_{k}$ during the first three cycles.

aggregate energy consumption rate for transmission at node $i$ is $\sum_{j \in \mathcal{N}}^{j \neq i} C_{i j} \cdot f_{i j}+C_{i B} \cdot f_{i B}$.

The energy consumption rate for reception at node $i$ is modeled as $\rho \sum_{k \in \mathcal{N}}^{k \neq i} f_{k i}$, where $\rho$ is the rate of energy consumption for receiving one unit of data rate.

Denote $p_{i}$ as the energy consumption rate at sensor node $i \in$ $\mathcal{N}$, which includes energy consumption both for transmission and reception. We have

$$
p_{i}=\rho \sum_{k \in \mathcal{N}}^{k \neq i} f_{k i}+\sum_{j \in \mathcal{N}}^{j \neq i} C_{i j} f_{i j}+C_{i B} f_{i B} \quad(i \in \mathcal{N}) .
$$

\section{Energy Dynamics at a Sensor Node}

In Section II-B, we discussed the behavior of WCV over a cycle time of $\tau$, during which the WCV starts from the service station, travels to those cells that have sensor nodes, and returns to the service station (see Fig. 3).

When the WCV charges sensor nodes in a cell, each node in the same cell usually does not reach $E_{\max }$ at the same time. So a node that reaches $E_{\max }$ before the WCV departs the cell will be in a "saturation" state where its battery will remain at $E_{\max }$ until the WCV departs this cell (see Fig. 4).

We now develop constraints to capture the saturation phenomena while ensuring that the energy level of each node never falls below $E_{\min }$. Denote $e_{i}(t)$ as node $i$ 's energy level at time $t$. The energy curve of node $i \in \mathcal{N}_{k}$ in a cell $k$ for the first three cycles is shown in Fig. 4. For any cycle, we see that there can be only three possible slopes: (i) a slope of $-p_{i}$ when the WCV is not in node $i$ 's cell, (ii) a slope of $\left(U_{i}-p_{i}\right)$ when the WCV is at node $i$ 's cell and is charging node $i$ at rate $U_{i},{ }^{3}$ and (iii) a slope of 0 (i.e., saturation period) when node $i$ stays at $E_{\max }$ while the WCV is still charging.

Denote $a_{k}$ as the arrival time of the WCV at cell $k$ in the first cycle. Denote $D_{\pi_{0} \pi_{1}}$ the distance between the service station and the first cell visited along $P$ and $D_{\pi_{l} \pi_{l+1}}$, the distance between the $l$ th and $(l+1)$ th cells, respectively. Then we have

$$
a_{\pi_{k}}=\sum_{l=0}^{k-1} \frac{D_{\pi_{l} \pi_{l+1}}}{V}+\sum_{l=1}^{k-1} \tau_{\pi_{l}}, k=1,2, \ldots,|\mathcal{Q}| .
$$

${ }^{3}$ Note that it is necessary to have $U_{i} \geq p_{i}, i \in \mathcal{N}$, to achieve a feasible solution. 
Note that $e_{i}\left(m \tau+a_{k}\right), m \in \mathbb{N}$, is a local minimum for $e_{i}(t)$. To have $e_{i}(t) \geq E_{\min }$ for all $t \geq 0$, it is sufficient to have

$$
e_{i}\left(m \tau+a_{k}\right)=e_{i}(m \tau)-a_{k} \cdot p_{i} \geq E_{\min }
$$

for all $m \in \mathbb{N}, i \in \mathcal{N}_{k}, k \in \mathcal{Q}$.

When $m=0, e_{i}\left(a_{k}\right)=e_{i}(0)-a_{k} \cdot p_{i}=E_{\max }-a_{k} \cdot p_{i}$. For $e_{i}\left(a_{k}\right) \geq E_{\min }$, we must have

$$
E_{\max }-a_{k} \cdot p_{i} \geq E_{\min } \quad\left(i \in \mathcal{N}_{k}, k \in \mathcal{Q}\right) .
$$

When $m \geq 1$,

$$
\begin{aligned}
e_{i}\left(m \tau+a_{k}\right)= & e_{i}(m \tau)-a_{k} \cdot p_{i} \\
= & e_{i}\left((m-1) \tau+a_{k}+\tau_{k}\right) \\
& -\left\{m \tau-\left[(m-1) \tau+a_{k}+\tau_{k}\right]\right\} \cdot p_{i} \\
& -a_{k} \cdot p_{i} \\
= & e_{i}\left((m-1) \tau+a_{k}+\tau_{k}\right)-\left(\tau-\tau_{k}\right) \cdot p_{i} \\
\leq & E_{\max }-\left(\tau-\tau_{k}\right) \cdot p_{i},
\end{aligned}
$$

where the last inequality holds since $e_{i}$ cannot exceed $E_{\max }$. For (6), if $e_{i}\left(m \tau+a_{k}\right) \geq E_{\min }$ for all $m \geq 1$, then we must have

$$
E_{\max }-\left(\tau-\tau_{k}\right) \cdot p_{i} \geq E_{\min } \quad\left(i \in \mathcal{N}_{k}, k \in \mathcal{Q}\right) .
$$

Now we show that once (7) holds, (5) must also hold. Therefore, we can remove (5) in the formulation. To see this, we have $a_{k}+\tau_{k}<\tau$, which leads to $E_{\max }-a_{k} \cdot p_{i}>$ $E_{\max }-\left(\tau-\tau_{k}\right) \cdot p_{i}$

Note that (7) is a necessary condition for $e_{i}(t) \geq E_{\min }$. The following is a second necessary condition for $e_{i}(t) \geq E_{\min }$.

$$
\tau \cdot p_{i}-U_{i} \cdot \tau_{k} \leq 0 \quad\left(i \in \mathcal{N}_{k}, k \in \mathcal{Q}\right),
$$

which says that the amount of energy being charged to node $i \in \mathcal{N}_{k}$ during the time period of $\tau_{k}, U_{i} \cdot \tau_{k}$, must be greater than or equal to the amount of energy consumed during the cycle, $\tau \cdot p_{i}$. (8) can be easily proved by showing that if $\tau$. $p_{i}-U_{i} \cdot \tau_{k}>0$, then $e_{i}(t)$ will fall below $E_{\text {min }}$ eventually at some time $t$.

We have shown that (7) and (8) are necessary conditions for $e_{i}(t) \geq E_{\min }$. It turns out that they are also sufficient conditions. We state this result in the following lemma.

Lemma 1: $e_{i}(t) \geq E_{\text {min }}$ for all $t \geq 0, i \in \mathcal{N}$, if and only if both constraints (7) and (8) are satisfied.

The "only if" part of the lemma (i.e., (7) and (8) are necessary conditions) was already proved in earlier discussion. We only need to prove the "if" part of the lemma. Recall that, to have $e_{i}(t) \geq E_{\min }$, it is sufficient to have $e_{i}\left(m \tau+a_{k}\right) \geq E_{\min }$, for $m \in \mathbb{N}, i \in \mathcal{N}$. Therefore, we can show that if (7) and (8) hold, $e_{i}\left(m \tau+a_{k}\right) \geq E_{\min }$, for $m \in \mathbb{N}, i \in \mathcal{N}$. We omit the formal proof here due to space limit and refer readers to [21] for details. The following corollary follows from the proof of Lemma 1.

Corollary 1.1: When the WCV departs cell $k, k \in \mathcal{Q}$, each sensor node $i \in \mathcal{N}_{k}$ is fully charged to $E_{\text {max }}$.

\section{Problem Formulation AND Properties}

Based on the constraints that we have discussed in Section II, a number of optimization problems can be formulated. One performance objective is to maximizing the ratio of $\frac{\tau_{\mathrm{vac}}}{\tau}$, which is a measure of the percentage of time the WCV is in vacation over its cycle time. A plausible goal is to maximize this ratio so that the $\mathrm{WCV}$ can spend most percentage of time on vacation (or equivalently, least percentage of time in the field). Mathematically, this is also a very challenging objective, as it involves a ratio of two variables. Therefore, a successful solution to this optimization problem will help pave the way to solve many other optimization problems with simpler objectives.

We now summarize our optimization problem as follows.

$$
\begin{array}{cll}
\max & \frac{\tau_{\mathrm{vac}}}{\tau} & \\
\text { s.t. } & (1),(2),(3),(7) \text { and }(8) & \\
& \tau, \tau_{P}, \tau_{\mathrm{vac}}, \tau_{k}, f_{i j}, f_{i B} \geq 0 & (i, j \in \mathcal{N}, i \neq j) \\
0 \leq p_{i} \leq U_{i} & (i \in \mathcal{N})
\end{array}
$$

In this problem, time intervals $\tau, \tau_{P}, \tau_{\text {vac }}$ and $\tau_{k}$, flow rates $f_{i j}$ and $f_{i B}$, and power consumption rate $p_{i}$ are optimization variables; $R_{i}, \rho, C_{i j}, C_{i B}, U_{i}, E_{\max }$, and $E_{\min }$ are constants. Note that $\tau_{P}$ can be determined once the traveling path $P$ is determined.

This problem is a nonlinear program (NLP), with nonlinear objective $\left(\frac{\tau_{\mathrm{vac}}}{\tau}\right)$ and nonlinear terms $\left(\tau \cdot p_{i}\right.$ and $\left.\tau_{k} \cdot p_{i}\right)$ in constraints (7) and (8). An NLP is NP-hard in general. Nevertheless, we can still find several useful properties associated with an optimal solution.

Property 1: In an optimal solution with the maximal $\frac{\tau_{\mathrm{vac}}}{\tau}$, the WCV must move along the shortest Hamiltonian cycle that connects the service station and the centers of cells $k \in \mathcal{Q}$. If the shortest Hamiltonian cycle is not unique, then any shortest Hamiltonian cycle can achieve the same optimal objective. Further, the WCV can follow either clockwise or counterclockwise direction of the shortest Hamiltonian cycle, both of which will achieve the same optimal objective.

A proof of this property can be given based on contradiction and shares a similar idea to a proof in [17].

The shortest Hamiltonian cycle can be obtained by solving the well known Traveling Salesman Problem (TSP) [1], [3]. Denote $D_{\text {TSP }}$ as the total path distance for the shortest Hamiltonian cycle and $\tau_{\mathrm{TSP}}=D_{\mathrm{TSP}} / V$. Then (1) becomes

$$
\tau-\sum_{k \in \mathcal{Q}} \tau_{k}-\tau_{\text {vac }}=\tau_{\text {TSP }} .
$$

For (9), we divide both sides by $\tau$ and have $1-\sum_{k \in \mathcal{Q}} \frac{\tau_{k}}{\tau}-$ $\frac{\tau_{\mathrm{vac}}}{\tau}=\tau_{\mathrm{TSP}} \cdot \frac{1}{\tau}$. We define $\eta_{\mathrm{vac}}=\frac{\tau_{\mathrm{vac}}}{\tau}$, which is our objective function in the optimization problem. Similarly, we define $\eta_{k}=\frac{\tau_{k}}{\tau}$, for $k \in \mathcal{Q}$, and $h=\frac{1}{\tau}$, where $\eta_{k}$ represents the ratio of the charging time at cell $k$ to the entire cycle time. Then (9) is written as $1-\sum_{k \in \mathcal{Q}} \eta_{k}-\eta_{\text {vac }}=\tau_{\text {TSP }} \cdot h$, or equivalently, $h=\frac{1-\sum_{k \in \mathcal{Q}} \eta_{k}-\eta_{\mathrm{vac}}}{\tau_{\mathrm{TSP}}}$. 


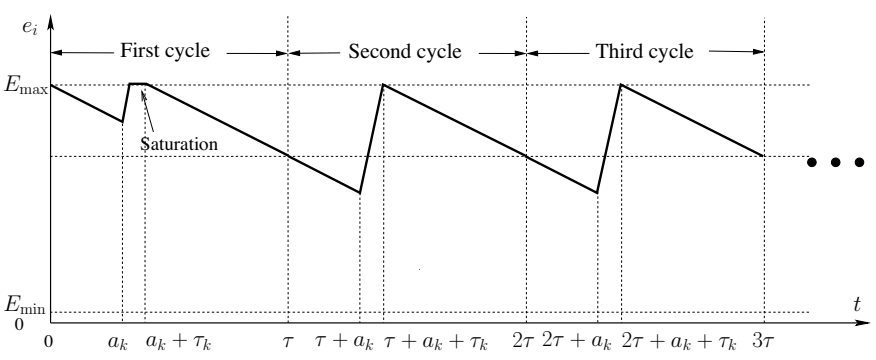

Fig. 5. The energy level of an equilibrium node $i \in \mathcal{N}_{k}$ in the first three cycles.

Similarly, by dividing both sides by $\tau$, replacing $\frac{\tau_{k}}{\tau}$ with $\eta_{k}$, and replacing $\frac{1}{\tau}$ with $\frac{1-\sum_{k \in \mathcal{Q}} \eta_{k}-\eta_{\mathrm{vac}}}{\tau_{\mathrm{TSP}}}$, (7) and (8) can be reformulated as,

$$
\begin{aligned}
\left(1-\eta_{k}\right) \cdot p_{i} \leq\left(E_{\max }-E_{\min }\right) \cdot \frac{1-\sum_{j \in \mathcal{Q}} \eta_{j}-\eta_{\mathrm{vac}}}{\tau_{\text {TSP }}} & \\
p_{i}-U_{i} \cdot \eta_{k} \leq 0 & \left(i \in \mathcal{N}_{k}, k \in \mathcal{Q}\right),
\end{aligned}
$$

We can rewrite (10) as

$\eta_{\text {vac }} \leq 1-\sum_{j \in \mathcal{Q}} \eta_{j}-\frac{\tau_{\mathrm{TSP}}}{E_{\max }-E_{\min }}\left(1-\eta_{k}\right) \cdot p_{i} \quad\left(i \in \mathcal{N}_{k}, k \in \mathcal{Q}\right)$.

Now our problem is reformulated as follows.

\section{OPT}

$$
\begin{aligned}
& \max \quad \eta_{\text {vac }} \\
& \text { s.t. } \quad \sum_{j \in \mathcal{N}}^{j \neq i} f_{i j}+f_{i B}-\sum_{k \in \mathcal{N}}^{k \neq i} f_{k i}=R_{i} \quad(i \in \mathcal{N}) \\
& \rho \cdot \sum_{k \in \mathcal{N}}^{k \neq i} f_{k i}+\sum_{j \in \mathcal{N}}^{j \neq i} C_{i j} \cdot f_{i j}+C_{i B} \cdot f_{i B}-p_{i}=0 \\
& (i \in \mathcal{N}) \\
& \eta_{\mathrm{vac}} \leq 1-\sum_{j \in \mathcal{Q}} \eta_{j}-\frac{\tau_{\mathrm{TSP}}}{E_{\max }-E_{\min }}\left(1-\eta_{k}\right) \cdot p_{i} \\
& \left(i \in \mathcal{N}_{k}, k \in \mathcal{Q}\right) \\
& p_{i}-U_{i} \cdot \eta_{k} \leq 0 \quad\left(i \in \mathcal{N}_{k}, k \in \mathcal{Q}\right) \\
& f_{i j}, f_{i B} \geq 0,0 \leq p_{i} \leq U_{i}, 0 \leq \eta_{k}, \eta_{\text {vac }} \leq 1 \\
& (i, j \in \mathcal{N}, i \neq j)
\end{aligned}
$$

In this problem, $f_{i j}, f_{i B}, p_{i}, \eta_{\text {vac }}$, and $\eta_{k}$ are optimization variables; $R_{i}, \rho, C_{i j}, C_{i B}, U_{i}, E_{\max }, E_{\min }$ and $\tau_{\mathrm{TSP}}$ are constants. Once we obtain a solution to problem OPT, we can recover $\tau, \tau_{k}$, and $\tau_{\text {vac }}$ as follows:

$$
\begin{aligned}
\tau & =\frac{\tau_{\mathrm{TSP}}}{1-\sum_{k \in \mathcal{Q}} \eta_{k}-\eta_{\mathrm{vac}}}, \\
\tau_{k} & =\tau \cdot \eta_{k}, \\
\tau_{\mathrm{vac}} & =\tau \cdot \eta_{\mathrm{vac}} .
\end{aligned}
$$

Based on Corollary 1.1, we know that when the WCV departs a cell $k, k \in \mathcal{Q}$, each sensor node in this cell is fully charged to $E_{\max }$. Further, some nodes may experience saturation state at every cycle. The following property says that in an optimal solution, at least one sensor node in each cell $k \in \mathcal{Q}$ will have saturation-free cycles except its initial first cycle (see Fig. 5)

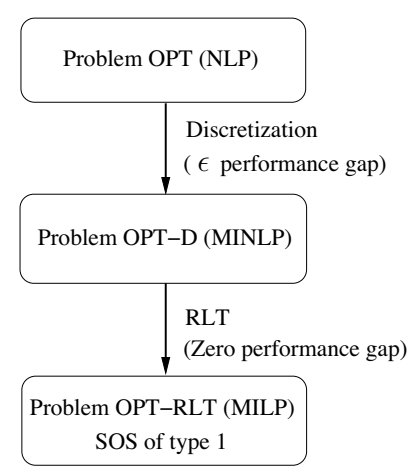

Fig. 6. A flow chart of our solution roadmap.

Property 2: In an optimal solution to OPT, there exists at least one node in each cell $k \in \mathcal{Q}$ such that, starting from the second cycle, the amount of energy reception at the node is the same as that of the energy consumption in each cycle.

We call such node in Property 2 equilibrium node. Note that the definition of equilibrium node is different from the so-called bottleneck node in [17], which is defined as the node whose energy level drops exactly to $E_{\min }$ upon WCV's arrival.

Property 2 can be proved by contradiction. That is, suppose that there exists an optimal solution to problem OPT that has no equilibrium node in some cell. We can always construct a new solution which increases the objective value, thus leading to contradiction. The formal proof of Property 2 is given in [21].

\section{A Near-Optimal Solution}

\section{A. Approach}

Problem OPT is a nonlinear program (NLP), with bilinear terms $\left(\eta_{k} \cdot p_{i}\right)$ in constraints (11). This nonlinear (bilinear) program is nonconvex [4], and cannot be solved by existing off-the-shelf solvers.

In this section, we convert the NLP to a mixed-integer linear program (MILP), which can then be solved efficiently by an off-the-shelf solver such as CPLEX [8]. First, we discretize variable $\eta_{k}$ in the bilinear term $\eta_{k} \cdot p_{i}$ using binary variables. This converts problem OPT to a $0-1$ mixed-integer nonlinear program (MINLP). By exploiting the special structures of the 0-1 MINLP, we employ a powerful technique called Reformulation-Linearization Technique (RLT) [18] to eliminate all bilinear terms. Subsequently, we have a 0-1 MILP, and we show that this new 0-1 MILP and the 0-1 MINLP have zero performance gap. This MILP has special ordered sets (SOS), which can be efficiently solved by CPLEX solver [8]. We quantify performance gap (due to discretization) and prove near-optimality of our solution. A flow chart of our solution roadmap is shown in Fig. 6.

\section{B. Solution Details}

1) Discretization: As a first step to reformulate the NLP to MILP, we consider the bilinear term $\eta_{k} \cdot p_{i}$. Since $\eta_{k}$ is 
a continuous variable within $[0,1]$, we discretize it by using $L+1$ discrete points $\eta_{k l}=\frac{l}{L}, l=0,1, \cdots, L$. Then we write

$$
\begin{array}{cc}
\eta_{k}=\sum_{l=0}^{L} \eta_{k l} \cdot z_{k l} & k \in \mathcal{Q}, \\
\sum_{l=0}^{L} z_{k l}=1 & k \in \mathcal{Q},
\end{array}
$$

where $z_{k l}$ is a binary variable that indicates whether or not $\eta_{k l}$ is chosen. By (16), the term $\eta_{k} \cdot p_{i}$ in (11) can be rewritten as $\eta_{k} \cdot p_{i}=\sum_{l=0}^{L} \eta_{k l} \cdot z_{k l} \cdot p_{i}$, which remains a bilinear term involving binary variables $z_{k l}, l=1,2, \cdots, L$. This makes the problem a 0-1 MINLP, which is formulated as follows.

\section{OPT-D}

$$
\begin{gathered}
\eta_{\text {vac }} \\
\text { s.t. } \sum_{j \in \mathcal{N}}^{j \neq i} f_{i j}+f_{i B}-\sum_{k \in \mathcal{N}}^{k \neq i} f_{k i}=R_{i} \quad(i \in \mathcal{N}) \\
\rho \cdot \sum_{k \in \mathcal{N}}^{k \neq i} f_{k i}+\sum_{j \in \mathcal{N}}^{j \neq i} C_{i j} \cdot f_{i j}+C_{i B} \cdot f_{i B}-p_{i}=0 \\
(i \in \mathcal{N}) \\
\eta_{\text {vac }} \leq 1-\sum_{j \in \mathcal{Q}} \sum_{l=0}^{L} \eta_{j l} \cdot z_{j l}-\frac{\tau_{\text {TSP }}}{E_{\max }-E_{\min }} \\
\cdot\left(p_{i}-\sum_{l=0}^{L} \eta_{k l} \cdot z_{k l} \cdot p_{i}\right) \quad\left(i \in \mathcal{N}_{k}, k \in \mathcal{Q}\right)(18) \\
p_{i}-U_{i} \sum_{l=0}^{L} \eta_{k l} \cdot z_{k l} \leq 0 \quad\left(i \in \mathcal{N}_{k}, k \in \mathcal{Q}\right)(19) \\
\sum_{l=0}^{L} z_{k l}=1 \quad(k \in \mathcal{Q}) \\
f_{i j}, f_{i B} \geq 0,0 \leq p_{i} \leq U_{i}, 0 \leq \eta_{\mathrm{vac}} \leq 1 \quad(i, j \in \mathcal{N}, i \neq j) \\
z_{k l} \in\{0,1\} \quad\left(i \in \mathcal{N}_{k}, k \in \mathcal{Q}, l=0, \cdots, L\right) .
\end{gathered}
$$

2) Reformulation and Linearization: To remove the nonlinear terms $z_{k l} \cdot p_{i}$ in the 0-1 MINLP, we employ a powerful technique called Reformulation-Linearization Technique (RLT) [18] as follows. Define

$$
\gamma_{i k l} \equiv z_{k l} \cdot p_{i}, \quad i \in \mathcal{N}_{k}, k \in \mathcal{Q}, l=0, \cdots, L .
$$

Then $\sum_{l=0}^{L} \eta_{k l} \cdot z_{k l} \cdot p_{i}$ can be rewritten as $\sum_{l=0}^{L} \eta_{k l} \cdot z_{k l} \cdot p_{i}=$ $\sum_{l=0}^{L} \eta_{k l} \cdot \gamma_{i k l}, i \in \mathcal{N}_{k}, k \in \mathcal{Q}$.

To replace the nonlinear constraint (20), we need to add RLT constraints, which are linear. The new linear constraints are generated by multiplying existing linear constraints for variables $z_{k l}$ and $p_{i}$, which are $1-\sum_{l=0}^{L} z_{k l}=0, z_{k l} \geq 0, p_{i} \geq 0$ and $U_{i}-p_{i} \geq 0$. It is worth pointing out that RLT in [19] typically refers to multiplying each pair of these constraints (i.e., reformulation) and generating linear constraints via variable substitution (i.e., linearization). For our problem, this may produce several redundant or null constraints. To reduce such redundancy, we exploit a special structure of our problem, i.e., the presence of equality constraints $1-\sum_{l=0}^{L} z_{k l}=0$. We can multiply these equality constraints (and $z_{k l} \geq 0$ ) by $p_{i} \geq 0$ and $U_{i}-p_{i} \geq 0$. Multiplying $1-\sum_{l=0}^{L} z_{k l}=0$ by $p_{i} \geq 0$ gives us

$$
\sum_{l=0}^{L} z_{k l} \cdot p_{i}=p_{i} \quad i \in \mathcal{N}_{k}, k \in \mathcal{Q},
$$

which can be rewritten as

$$
\sum_{l=0}^{L} \gamma_{i k l}=p_{i} \quad i \in \mathcal{N}_{k}, k \in \mathcal{Q} .
$$

Multiplying $1-\sum_{l=0}^{L} z_{k l}=0$ by $U_{i}-p_{i} \geq 0$ simply produces constraint $(21)$, or $1-\sum_{l=0}^{L} z_{k l}=0$ if $U_{i}-p_{i}>0$, or $U_{i}-p_{i}=0$, none of which is new.

Multiplying $p_{i} \geq 0$ and $U_{i}-p_{i} \geq 0$ by $z_{k l} \geq 0$, respectively, we have the following RLT constraints.

$$
0 \leq z_{k l} \cdot p_{i} \leq U_{i} z_{k l} \quad i \in \mathcal{N}_{k}, k \in \mathcal{Q}, l=0, \cdots, L,
$$

which can be rewritten as

$$
0 \leq \gamma_{i k l} \leq U_{i} z_{k l} \quad i \in \mathcal{N}_{k}, k \in \mathcal{Q}, l=0, \cdots, L .
$$

In summary, the new RLT constraints are (21) and (22). By substituting (20) for $z_{k l} \cdot p_{i}$, and adding the new RLT constraints (21) and (22), we obtain the following 0-1 MILP.

$$
\begin{aligned}
& \text { OPT-RLT } \\
& \max \quad \eta_{\text {vac }} \\
& \text { s.t. } \quad \sum_{j \in \mathcal{N}}^{j \neq i} f_{i j}+f_{i B}-\sum_{k \in \mathcal{N}}^{k \neq i} f_{k i}=R_{i} \quad(i \in \mathcal{N}) \\
& \rho \cdot \sum_{k \in \mathcal{N}}^{k \neq i} f_{k i}+\sum_{j \in \mathcal{N}}^{j \neq i} C_{i j} \cdot f_{i j}+C_{i B} \cdot f_{i B}-p_{i}=0 \\
& (i \in \mathcal{N}) \\
& \eta_{\mathrm{vac}} \leq 1-\sum_{j \in \mathcal{Q}} \sum_{l=0}^{L} \eta_{j l} \cdot z_{j l}-\frac{\tau_{\mathrm{TSP}}}{E_{\max }-E_{\min }} \\
& \cdot\left(p_{i}-\sum_{l=0}^{L} \eta_{k l} \cdot \gamma_{i k l}\right) \quad\left(i \in \mathcal{N}_{k}, k \in \mathcal{Q}\right) \\
& p_{i}-U_{i} \sum_{l=0}^{L} \eta_{k l} \cdot z_{k l} \leq 0 \quad\left(i \in \mathcal{N}_{k}, k \in \mathcal{Q}\right) \\
& \sum_{l=0}^{L} z_{k l}=1 \\
& \sum_{l=0}^{L} \gamma_{i k l}-p_{i}=0 \quad\left(i \in \mathcal{N}_{k}, k \in \mathcal{Q}\right) \\
& \gamma_{i k l}-U_{i} z_{k l} \leq 0 \quad\left(i \in \mathcal{N}_{k}, k \in \mathcal{Q}, l=0, \cdots, L\right) \\
& f_{i j}, f_{i B} \geq 0,0 \leq p_{i} \leq U_{i}, 0 \leq \eta_{\text {vac }} \leq 1 \quad(i, j \in \mathcal{N}, i \neq j) \\
& \gamma_{i k l} \geq 0, z_{k l} \in\{0,1\}\left(i \in \mathcal{N}_{k}, k \in \mathcal{Q}, l=0, \cdots, L\right) .
\end{aligned}
$$

In this problem, $f_{i j}, f_{i B}, p_{i}, \eta_{\mathrm{vac}}$, and $\gamma_{i k l}$ are continuous variables; $z_{k l}$ are binary variables; $R_{i}, \rho, C_{i j}, C_{i B}, U_{i}, \eta_{k l}$, $E_{\max }, E_{\min }$, and $\tau_{\mathrm{TSP}}$ are constants. The integer variables $z_{k l}$, $l=0,1, \cdots, L$, are constrained by (17) and form a special ordered set (SOS) of type 1 (meaning that at most one of the variables in the set may be non-zero) [2]. It turns out that such special type of MILP is particularly suitable for CPLEX solve as CPLEX can use special branching strategies to improve performance [8].

Through RLT, we have eliminated all bilinear terms in the 0-1 MINLP and have obtained a 0-1 MILP. A natural question to ask is how much the performance gap between the optimal solutions under MINLP and MILP is. The following lemma says that the performance gap between the two is zero, thus substantiating the benefits of employing RLT in our solution approach. By zero performance gap, we mean there is a bijection from the feasible region of problem OPT-D to the feasible region of problem OPT-RLT and vice versa; and any two feasible solutions corresponding to this one-to-one mapping achieve the same objective value.

Lemma 2: Problem OPT-RLT and problem OPT-D have zero performance gap.

Our proof consists of two parts. (i) If a solution $\psi_{\mathrm{D}}=\left(f_{i j}\right.$, $\left.f_{i B}, p_{i}, \eta_{\text {vac }}, z_{k l}\right)$ is feasible to problem OPT-D, then the 
solution $\psi_{\mathrm{RLT}}=\left(f_{i j}, f_{i B}, p_{i}, \eta_{\mathrm{vac}}, z_{k l}, \gamma_{i k l}\right)$ is also feasible to problem OPT-RLT, where $\gamma_{i k l}=p_{i} \cdot z_{k l}$. (ii) If a solution $\psi_{\mathrm{RLT}}=\left(f_{i j}, f_{i B}, p_{i}, \eta_{\mathrm{vac}}, z_{k l}, \gamma_{i k l}\right)$ is feasible to problem OPT-RLT, then the solution $\psi_{\mathrm{D}}=\left(f_{i j}, f_{i B}, p_{i}, \eta_{\mathrm{vac}}, z_{k l}\right)$ is also feasible to problem OPT-D. If we can prove both (i) and (ii) are true, then there is a bijection from the feasible region of problem OPT-D to the feasible region of problem OPTRLT and vice verse; and for any one-to-one solution mapping between $\psi_{\mathrm{D}}$ and $\psi_{\mathrm{RLT}}$, their objective values are the same. A formal proof of Lemma 2 is given in [21].

3) Recovering a Solution to the Original Problem: By now, we have obtained a solvable 0-1 MILP. Once we have an optimal solution to this MILP, the question to ask is how to recover a feasible solution to the original problem (OPT). Assuming we have a solution $\psi_{\mathrm{RLT}}=\left(f_{i j}, f_{i B}, p_{i}, \eta_{\mathrm{vac}}, z_{k l}, \gamma_{i k l}\right)$ that is optimal to problem OPT-RLT, by Lemma 2, the solution $\psi_{\mathrm{D}}=\left(f_{i j}, f_{i B}, p_{i}, \eta_{\mathrm{vac}}, z_{k l}\right)$ is also feasible to problem OPT-D. Based on $\psi_{\mathrm{D}}$, we can construct a solution $\psi=\left(f_{i j}, f_{i B}, p_{i}, \eta_{\mathrm{vac}}, \eta_{k}\right)$ to problem OPT by letting $\eta_{k}=$ $\sum_{l=0}^{L} \eta_{k l} \cdot z_{k l}$, and $f_{i j}, f_{i B}, p_{i}$ and $\eta_{\mathrm{vac}}$ unchanged from $\psi_{\mathrm{D}}$. Note that $\psi$ is a feasible solution to problem OPT since the constraints in problem OPT are the same as those in problem OPT-D after we replace $\eta_{k}$ by $\sum_{l=0}^{L} \eta_{k l} \cdot z_{k l}$. Since $\psi$ is only a feasible solution to problem OPT, its objective value $\eta_{\text {vac }}$ is a lower bound for problem OPT. We summarize our discussion in the following lemma.

Lemma 3: For a given optimal solution $\left(f_{i j}, f_{i B}, p_{i}, \eta_{\mathrm{vac}}\right.$, $\left.z_{k l}, \gamma_{i k l}\right)$ to problem OPT-RLT, we can construct a solution $\left(f_{i j}, f_{i B}, p_{i}, \eta_{\mathrm{vac}}, \eta_{k}\right)$ that is feasible to Problem OPT by letting $\eta_{k}=\sum_{l=0}^{L} \eta_{k l} \cdot z_{k l}$. Furthermore, $\eta_{\mathrm{vac}}$ is a lower bound for the optimal objective value of Problem OPT.

\section{Proof of Near-Optimality}

Recall that our original problem is OPT, which is a NLP. We converted this NLP to a 0-1 MINLP via discretization (problem OPT-D) and then to a 0-1 MILP via RLT (problem OPT-RLT). We proved that problem OPT-D and problemRLT have zero performance gap. So the performance gap between problems OPT and OPT-RLT could only occur during discretization.

Quantifying Performance Gap. By solving problem OPTRLT, we obtain an optimal solution $\psi_{\text {RLT }}$ to problem OPT-RLT. Denote $\psi^{*}$ the optimal (unknown) solution to problem OPT. Denote $\eta_{\text {vac }}$ the optimal objective value obtained by $\psi_{\mathrm{RLT}}$ and $\eta_{\text {vac }}^{*}$ the optimal objective value obtained by $\psi^{*}$, respectively. Naturally, the gap between $\eta_{\text {vac }}$ and $\eta_{\text {vac }}^{*}$ is closely related with $L$, which is the number of points used in discretization. We quantify this gap in the following lemma.

Lemma 4: For a given $L$, we have $\eta_{\mathrm{vac}}^{*}-\eta_{\mathrm{vac}} \leq \frac{|\mathcal{Q}|}{L}$.

In our proof, we consider two cases, depending on whether $\eta_{\text {vac }}^{*} \leq \frac{|\mathcal{Q}|}{L}$ or $\eta_{\text {vac }}^{*}>\frac{|\mathcal{Q}|}{L}$. The first case is trivial since $\eta_{\text {vac }} \geq$ 0 . The second case needs more elaborate effort and we refer readers to [21] for details.

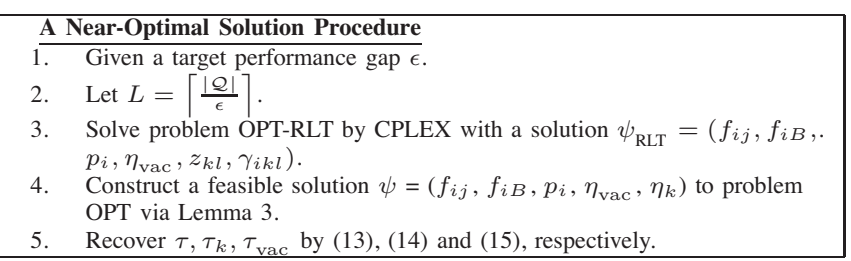

Fig. 7. A summary of the proposed near-optimal solution procedure.

Performance Guarantee. Lemma 4 gives an upper bound of the performance gap between $\eta_{\mathrm{vac}}$ and $\eta_{\mathrm{vac}}^{*}$ for a given $L$. The following lemma shows how to choose $L$ so that this performance gap is no more than $\epsilon(0<\epsilon \ll 1)$.

Lemma 5: For a given $\epsilon, 0<\epsilon \ll 1$, if $L=\left\lceil\frac{|\mathcal{Q}|}{\epsilon}\right\rceil$, we have $\eta_{\mathrm{vac}}^{*}-\eta_{\mathrm{vac}} \leq \epsilon$.

Proof: By Lemma 4, we know $\eta_{\text {vac }}^{*}-\eta_{\text {vac }} \leq \frac{|\mathcal{Q}|}{L}$. To have $\eta_{\text {vac }}^{*}-\eta_{\text {vac }} \leq \epsilon$, it is sufficient to have $\frac{|\mathcal{Q}|}{L} \leq \epsilon$, or $L=\left\lceil\frac{|\mathcal{Q}|}{\epsilon}\right\rceil$. This completes the proof.

We summarize our near-optimal solution procedure to OPT in Fig. 7.

\section{NumERICAL RESUltS}

In this section, we present some numerical results to demonstrate how our solution works to achieve multi-node wireless energy transfer.

\section{A. Simulation Settings}

We assume sensor nodes are deployed over a $1000 \mathrm{~m} \times$ $1000 \mathrm{~m}$ square area. The base station is at $(500,500)$ (in $\mathrm{m}$ ) and the WCV's home service station is assumed to be at the origin. The traveling speed of the WCV is $V=5 \mathrm{~m} / \mathrm{s}$. The data rate $R_{i}, i \in \mathcal{N}$, from each node is randomly generated within $[1,10] \mathrm{kb} / \mathrm{s}$. The power consumption coefficients are $\beta_{1}=50 \mathrm{~nJ} / \mathrm{b}, \beta_{2}=0.0013 \mathrm{pJ} /\left(\mathrm{b} \cdot \mathrm{m}^{4}\right)$, and $\rho=50 \mathrm{~nJ} / \mathrm{b}[5]$. The path loss index is $\alpha=4$.

For the battery at a sensor node, we choose a regular NiMH battery and its nominal cell voltage and volume of electricity is $1.2 \mathrm{~V} / 2.5 \mathrm{Ah}$. We have $E_{\max }=1.2 \mathrm{~V} \times 2.5 \mathrm{~A} \times 3600 \mathrm{sec}=$ $10.8 \mathrm{~kJ}$ [11]. We let $E_{\min }=0.05 \cdot E_{\max }=540 \mathrm{~J}$. For $\mu\left(D_{i}\right)$, we refer to the experimental data on wireless energy transfer efficiency in [10] (see Fig. 8). Through curve fitting, we obtain $\mu\left(D_{i}\right)=-0.0958 D_{i}^{2}-0.0377 D_{i}+1.0$. Assuming $U_{\text {Full }}=5 \mathrm{~W}$ and $\delta=1 \mathrm{~W}$, we have $D=2.7 \mathrm{~m}$ for a cell's side length. We set $\epsilon=0.1$ for the numerical results.

\section{B. Results of a 100-node Network}

We first present complete results for a 100-node network. Table I gives the location of each node and its data rate for the 100 -node network. These 100 nodes are distributed in $|\mathcal{Q}|=$ 32 selected cells and Table II gives the location of each cell as well as the number of sensor nodes it contains. The shortest Hamiltonian cycle that threads all cells $k \in \mathcal{Q}$ and the service station is found by the Concorde TSP solver [3], which is 


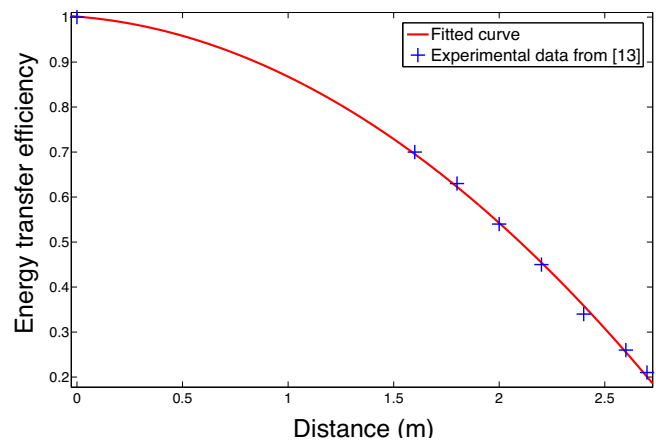

Fig. 8. Efficiency of energy transfer as a function of distance between a sensor node and the WCV. This figure is based on averaging energy transfer efficiencies for two devices in the experimental study in [10].

TABLE I

LOCATION AND DATA RATE $R_{i}$ FOR EACH NODE IN A 100 -NODE NETWORK

\begin{tabular}{|c|c|c|c|c|c|}
\hline $\begin{array}{l}\text { Node } \\
\text { index }\end{array}$ & $\begin{array}{l}\text { Location } \\
\text { (m) }\end{array}$ & $\begin{array}{c}R_{i} \\
(\mathrm{~Kb} / \mathrm{s})\end{array}$ & $\begin{array}{l}\text { Node } \\
\text { index }\end{array}$ & $\begin{array}{l}\text { Location } \\
\text { (m) }\end{array}$ & $\begin{array}{c}R_{i} \\
(\mathrm{~Kb} / \mathrm{s})\end{array}$ \\
\hline 1 & $(140.8,905.4)$ & 3 & 51 & $(613.9,474.1)$ & 9 \\
\hline 2 & $(977.8,913.0)$ & 5 & 52 & $(837.1,6.1)$ & 2 \\
\hline 3 & $(679.9,92.7)$ & 4 & 53 & $(635.8,216.4)$ & 4 \\
\hline 4 & $(325.8,378.1)$ & 5 & 54 & $(165.7,109.6)$ & 7 \\
\hline 5 & $(196.2,526.8)$ & 8 & 55 & $(634.9,218.4)$ & 1 \\
\hline 6 & $(546.5,967.0)$ & 2 & 56 & $(747.2,33.2)$ & 7 \\
\hline 7 & $(323.4,329.4)$ & 7 & 57 & $(821.4,608.6)$ & 5 \\
\hline 8 & $(747.7,33.4)$ & 3 & 58 & $(250.1,839.1)$ & 10 \\
\hline 9 & $(838.3,678.0)$ & 1 & 59 & $(990.6,617.0)$ & 6 \\
\hline 10 & $(692.5,461.4)$ & 9 & 60 & $(977.9,909.0)$ & 4 \\
\hline 11 & $(918.4,517.9)$ & 1 & 61 & $(585.6,623.4)$ & 5 \\
\hline 12 & $(613.6,476.1)$ & 1 & 62 & $(678.6,91.7)$ & 1 \\
\hline 13 & $(586.2,621.8)$ & 4 & 63 & $(613.5,475.9)$ & 1 \\
\hline 14 & $(440.2,17.7)$ & 4 & 64 & $(438.8,17.3)$ & 2 \\
\hline 15 & $(813.3,749.9)$ & 7 & 65 & $(836.5,680.4)$ & 10 \\
\hline 16 & $(886.2,612.7)$ & 7 & 66 & $(321.9,331.2)$ & 3 \\
\hline 17 & $(804.9,329.4)$ & 5 & 67 & $(752.3,714.6)$ & 9 \\
\hline 18 & $(633.7,218.4)$ & 4 & 68 & $(679.6,93.7)$ & 8 \\
\hline 19 & $(753.3,713.0)$ & 9 & 69 & $(838.0,677.4)$ & 6 \\
\hline 20 & $(163.7,108.6)$ & 2 & 70 & $(897.2,709.2)$ & 2 \\
\hline 21 & $(672.0,318.1)$ & 10 & 71 & $(750.8,712.4)$ & 3 \\
\hline 22 & $(250.2,840.1)$ & 1 & 72 & $(635.5,217.4)$ & 7 \\
\hline 23 & $(732.3,965.0)$ & 4 & 73 & $(585.4,622.0)$ & 6 \\
\hline 24 & $(197.4,672.2)$ & 6 & 74 & $(732.0,966.2)$ & 3 \\
\hline 25 & $(92.7,967.7)$ & 1 & 75 & $(91.5,971.3)$ & 2 \\
\hline 26 & $(990.1,617.4)$ & 4 & 76 & $(918.6,514.1)$ & 2 \\
\hline 27 & $(804.3,352.0)$ & 7 & 77 & $(166.0,109.0)$ & 8 \\
\hline 28 & $(821.5,609.8)$ & 7 & 78 & $(90.6,967.7)$ & 10 \\
\hline 29 & $(898.3,708.4)$ & 6 & 79 & $(813.4,749.7)$ & 1 \\
\hline 30 & $(688.4,59.1)$ & 3 & 80 & $(686.9,59.7)$ & 10 \\
\hline 31 & $(837.3,4.5)$ & 2 & 81 & $(587.1,622.4)$ & 2 \\
\hline 32 & $(451.2,135.5)$ & 1 & 82 & $(838.2,680.4)$ & 4 \\
\hline 33 & $(979.3,911.8)$ & 8 & 83 & $(249.7,842.5)$ & 3 \\
\hline 34 & $(678.9,93.3)$ & 8 & 84 & $(139.9,902.0)$ & 7 \\
\hline 35 & $(751.6,714.2)$ & 6 & 85 & $(691.4,459.4)$ & 3 \\
\hline 36 & $(633.8,217.0)$ & 9 & 86 & $(747.3,36.0)$ & 5 \\
\hline 37 & $(452.9,133.7)$ & 3 & 87 & $(803.9,327.0)$ & 7 \\
\hline 38 & $(633.6,217.8)$ & 1 & 88 & $(164.6,108.4)$ & 3 \\
\hline 39 & $(884.4,613.9)$ & 1 & 89 & $(197.8,670.4)$ & 5 \\
\hline 40 & $(197.1,523.2)$ & 6 & 90 & $(820.8,610.2)$ & 3 \\
\hline 41 & $(813.8,747.7)$ & 5 & 91 & $(140.8,904.6)$ & 9 \\
\hline 42 & $(804.1,326.6)$ & 4 & 92 & $(546.3,965.4)$ & 4 \\
\hline 43 & $(732.9,966.2)$ & 3 & 93 & $(886.8,613.1)$ & 8 \\
\hline 44 & $(690.1,460.2)$ & 7 & 94 & $(671.5,318.9)$ & 8 \\
\hline 45 & $(669.9,319.1)$ & 7 & 95 & $(248.7,842.9)$ & 4 \\
\hline 46 & $(195.9,670.2)$ & 10 & 96 & $(670.6,318.1)$ & 7 \\
\hline 47 & $(440.7,17.1)$ & 2 & 97 & $(613.3,477.1)$ & 9 \\
\hline 48 & $(670.2,319.3)$ & 10 & 98 & $(614.2,475.7)$ & 5 \\
\hline 49 & $(585.1,624.2)$ & 1 & 99 & $(452.2,133.1)$ & 7 \\
\hline 50 & $(896.5,708.4)$ & 8 & 100 & $(197.4,670.6)$ & 4 \\
\hline
\end{tabular}

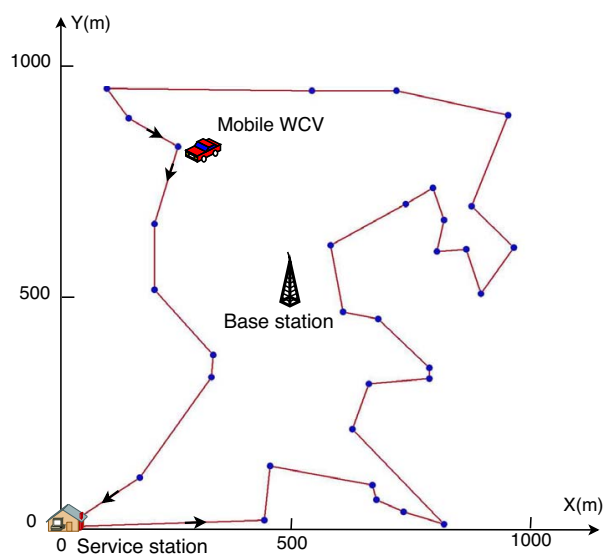

Fig. 9. An optimal traveling path (assuming counter clockwise direction) for the 100-node sensor network. The 100 nodes are distributed in 32 cells, with the center of each cell being represented as a point in the figure.

TABLE II

CELLS INDEX, LOCATION OF CELL CENTER, SENSOR NODES IN EACH CELL, CELL TRAVELING ORDER ALONG THE PATH, AND CHARGING TIME AT EACH CELL FOR THE 100-NODE NETWORK.

\begin{tabular}{|c|c|c|c|c|}
\hline $\begin{array}{l}\text { Cell } \\
\text { index }\end{array}$ & $\begin{array}{l}\text { Location of } \\
\text { cell center }(\mathrm{m})\end{array}$ & $\begin{array}{l}\text { Sensor nodes } \\
\text { in the cell }\end{array}$ & $\begin{array}{l}\text { Travel } \\
\text { order }\end{array}$ & $\begin{array}{l}\tau_{k} \\
(\mathrm{~s})\end{array}$ \\
\hline 1 & $(140.4,904.1)$ & $1,84,91$ & 26 & 157 \\
\hline 2 & $(452.3,134.8)$ & $32,37,99$ & 2 & 314 \\
\hline 3 & $(837.0,6.2)$ & 31,52 & 6 & 157 \\
\hline 4 & $(687.1,60.0)$ & 30,80 & 4 & 157 \\
\hline 5 & $(897.8,710.1)$ & $29,50,70$ & 21 & 157 \\
\hline 6 & $(820.8,609.5)$ & $28,57,90$ & 17 & 628 \\
\hline 7 & $(804.6,352.3)$ & 27 & 10 & 157 \\
\hline 8 & $(990.9,618.9)$ & 26,59 & 20 & 157 \\
\hline 9 & $(91.8,969.6)$ & $25,75,78$ & 25 & 157 \\
\hline 10 & $(197.1,670.3)$ & $24,46,89,100$ & 28 & 2510 \\
\hline 11 & $(731.7,964.9)$ & $23,43,74$ & 23 & 314 \\
\hline 12 & $(249.8,841.0)$ & $22,58,83,95$ & 27 & 471 \\
\hline 13 & $(670.9,317.2)$ & $21,45,48,94,96$ & 8 & 314 \\
\hline 14 & $(164.7,109.1)$ & $20,54,77,88$ & 32 & 471 \\
\hline 15 & $(751.9,714.7)$ & $19,35,67,71$ & 14 & 314 \\
\hline 16 & $(634.5,216.7)$ & $18,36,38,53,55,72$ & 7 & 157 \\
\hline 17 & $(804.6,328.9)$ & $17,42,87$ & 9 & 157 \\
\hline 18 & $(885.6,614.2)$ & $16,39,93$ & 18 & 157 \\
\hline 19 & $(812.7,749.8)$ & $15,41,79$ & 15 & 157 \\
\hline 20 & $(440.1,15.6)$ & $14,47,64$ & 1 & 157 \\
\hline 21 & $(585.9,623.5)$ & $13,49,61,73,81$ & 13 & 157 \\
\hline 22 & $(614.3,476.2)$ & $12,51,63,97,98$ & 12 & 314 \\
\hline 23 & $(918.0,516.0)$ & 11,76 & 19 & 157 \\
\hline 24 & $(691.2,459.9)$ & $10,44,85$ & 11 & 157 \\
\hline 25 & $(837.0,679.7)$ & $9,65,69,82$ & 16 & 157 \\
\hline 26 & $(747.9,34.3)$ & $8,56,86$ & 5 & 157 \\
\hline 27 & $(322.6,331.3)$ & 7,66 & 31 & 2353 \\
\hline 28 & $(545.4,964.9)$ & 6,92 & 24 & 157 \\
\hline 29 & $(197.1,525.3)$ & 5,40 & 29 & 784 \\
\hline 30 & $(326.7,380.4)$ & 4 & 30 & 157 \\
\hline 31 & $(679.0,92.8)$ & $3,34,62,68$ & 3 & 157 \\
\hline 32 & $(978.8,911.1)$ & $2,33,60$ & 22 & 314 \\
\hline
\end{tabular}

shown in Fig. 9. For this optimal cycle, $D_{\mathrm{TSP}}=5110 \mathrm{~m}$ and $\tau_{\text {TSP }}=1022 \mathrm{~s}$. For the target performance gap $\epsilon=0.1$, we have cycle time $\tau=13.95$ hours, vacation time $\tau_{\text {vac }}=10.26$ hours, and the objective $\eta_{\mathrm{vac}}=73.55 \%$.

Corollary 1.1 says that each sensor node in the network is fully charged to $E_{\max }$ when the WCV departs its cell, which is confirmed by our numerical results. Property 2 says that in an optimal solution, there exists at least one equilibrium node in each cell $k \in \mathcal{Q}$. In our numerical results, we find that all 32 cells contain equilibrium nodes. 


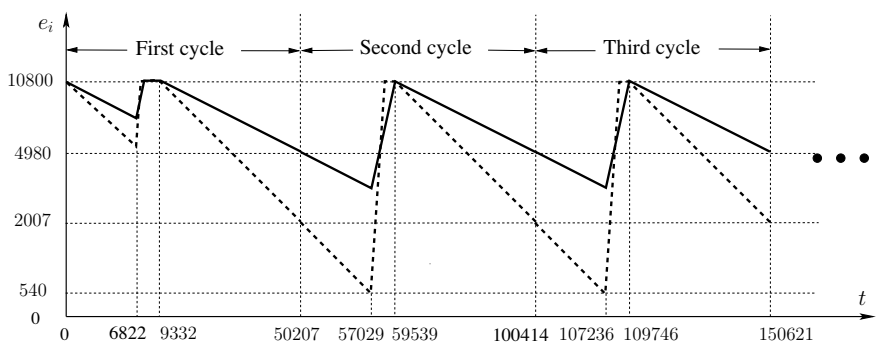

Fig. 10. Energy cycle behavior of an equilibrium node (node 24, in solid curve) and a non-equilibrium node (node 89 , in dashed curve) in the 100-node network.

To examine energy behavior at sensor nodes, consider sensor nodes in cell 10 . There are 4 sensor nodes in this cell, nodes 24 and 46 are equilibrium nodes while nodes 89 and 100 are not. Fig. 10 shows the energy behavior of node 24 (solid curve) and node 89 (dashed curve). Note that node 24 does not have any saturation period except in the initial first cycle while node 89 has saturation period in every cycle.

\section{CONCLUSION}

In this paper, we exploited recent advances in multi-node wireless energy transfer technology to charge the batteries of sensor nodes in a WSN. Our approach was to develop a formal optimization framework by jointly optimizing traveling path, flow routing and charging time at each cell. By employing discretization and a novel reformulation-linearization technique, we developed a provably near-optimal solution for any desired level of accuracy. Using numerical results, we demonstrated the capability of multi-node wireless energy transfer technology in addressing the scalability problem in a WSN.

\section{ACKNOWLEDGMENTS}

This work was supported in part by the NSF under grants ECCS-0925719 (Y.T. Hou and H.D. Sherali), CNS-1156311 (W. Lou), CNS-1156318 (W. Lou), and CMMI-0969169 (H.D. Sherali).

\section{REFERENCES}

[1] D. L. Applegate, R. E. Bixby, V. Chvatal, and W. J. Cook, The Traveling Salesman Problem: A Computational Study, Chapter 4, Princeton Univ. Press, Princeton, NJ, Jan. 2007.
[2] E. M. L. Beale and J. J. H. Forrest, "Global optimization using special ordered sets," Mathematical Programming, vol. 10, no. 1, pp. 52-69, 1976.

[3] Concorde TSP Solver, http://www.tsp.gatech.edu/concorde/.

[4] C.A. Floudas, Deterministic Global Optimization: Theory, Methods, and Applications, Chapter 2, Kluwer Academic, Dec. 1999.

[5] W.B. Heinzelman, "Application-specific protocol architectures for wireless networks," Ph.D. dissertation, Dept. Elect. Eng. Comput. Sci., MIT, Cambridge, MA, Jun. 2000.

[6] R. Horst and H. Tuy, Global Optimization: Deterministic Approaches, Chapter 4, 3rd edition, Springer-Verlag, 1996.

[7] Y.T. Hou, Y. Shi, and H.D. Sherali, "Rate allocation and network lifetime problems for wireless sensor networks," IEEE/ACM Trans. on Networking, vol. 16, no. 2, pp. 321-334, Apr. 2008

[8] IBM ILOG CPLEX Optimizer, http://www-01.ibm.com/software/integ ration/optimization/cplex-optimizer/.

[9] A. Kurs, A. Karalis, R. Moffatt, J.D. Joannopoulos, P. Fisher, and M. Soljacic, "Wireless power transfer via strongly coupled magnetic resonances," Science, vol. 317, no. 5834, pp. 83-86, July 2007.

[10] A. Kurs, R. Moffatt, and M. Soljacic, "Simultaneous mid-range power transfer to multiple devices," Appl. Phys. Lett., vol. 96, no. 4, article 4102, Jan. 2010.

[11] D. Linden and T.B. Reddy (eds.), Handbook of Batteries, 3rd ed., Chapter 1, McGraw-Hill, 2002.

[12] J. Messina, "Haier exhibits a wireless HDTV video system at the 2010 CES," URL: www.physorg.comnews $182608923 . \mathrm{html}$.

[13] G. L. Nemhauser and L. A. Wolsey, Integer and Combinatorial Optimization, Chapter II-4, John Wiley \& Sons Inc., Hoboken, NJ, July 1999.

[14] PowerCast, http://www.powercastco.com.

[15] A.K. RamRakhyani, S. Mirabbasi, and M. Chiao, "Design and optimization of resonance-based efficient wireless power delivery systems for biomedical implants," IEEE Trans. on Biomedical Circuits and Systems, vol. 5, no. 1, pp. 48-63, Feb. 2011.

[16] A. Sample, D. Yaniel, P. Powledge, A. Mamishev, and J. Smith, "Design of an rfid-based battery-free programmable sensing platform," IEEE Trans. on Instrumentation and Measurement, vol. 57, no. 11, pp. 26082615, Nov. 2008

[17] Y. Shi, L. Xie, Y.T. Hou, and H.D. Sherali, "On renewable sensor networks with wireless energy transfer," in Proc. IEEE INFOCOM, Shanghai, China, Apr. 2011, pp. 1350-1358.

[18] H.D. Sherali, W.P. Adams and P.J. Driscoll, "Exploiting special structures in constructing a hierarchy of relaxations for 0-1 mixed integer problems," Operations Research, vol. 46, no. 3, pp. 396-405, MayJune 1998.

[19] H.D. Sherali and W.P. Adams, A Reformulation- Linearization Technique for Solving Discrete and Continuous Nonconvex Problems, Kluwer Academic Publishers, Chapter 8, 1999.

[20] H. Tuy, Convex Analysis and Global Optimization, Chapter 5, Kluwer Academic, 1998.

[21] L. Xie, Y. Shi, Y.T. Hou, W. Lou, H.D. Sherali, and S. F. Midkiff, "On renewable sensor networks with wireless energy transfer: The MultiNode Case," Technical Report, the Bradley Department of Electrical and Computer Engineering, Virginia Tech, Blacksburg, VA, July 2011. Available at http://filebox.vt.edu/users/windgoon/papers/multicharging.pdf.

[22] F. Zhang, X. Liu, S.A. Hackworth, R.J. Sclabassi, and M. Sun, "In vitro and in vivo studies on wireless powering of medical sensors and implantable devices," in Proc. IEEE/NIH Life Science Systems and Applications Workshop (LiSSA), Bethesda, MD, Apr. 2009, pp. 84-87. 